\title{
Comment on: Adopting a palliative approach to dementia care in the community: a participatory action research
}

Singapore Med J 2021; 62(10): 560 https://doi.org/10.11622/smedj.2021204

Dear Sir,

I read with great interest the article 'Adopting a palliative approach to dementia care in the community: a participatory action research' by Tan et al. ${ }^{(1)}$

According to Population Trends 2019 from the Singapore Department of Statistics, the prevalence of dementia as of June 2019 was 86,050 for our population of 5.7 million. ${ }^{(2)}$ The Well-Being of the Singapore Elderly Study 2013 showed that one in ten persons aged above 60 years has dementia. ${ }^{(3)}$ In the report on Dementia Innovation Readiness Index 2020, Singapore ranked 16 th among 30 cities in the world. In the five domains of readiness assessed, Singapore had low scores in the domains of early detection and diagnosis. ${ }^{(4)}$

To combat the rising tide of dementia, early diagnosis is crucial not only for early treatment and implementation of strategies to slow the decline, but also because it affords time for engaging persons with dementia (PwDs) and their families to gradually accept that dementia is a terminal illness that will eventually require palliative care. While various dementia staging tools are available, the dual problems of great heterogeneity in the progression of dementia and difficulty predicting the stage when palliative care is required pose challenges for both family caregivers and healthcare professionals. ${ }^{(5)}$

Barriers to providing palliative care for dementia include poor recognition of the fact that dementia is a terminal illness; a protracted course with variable illness duration, unlike cancers; lack of capacity among PwDs in an advanced stage to convey their needs and wishes; lack of knowledge and skills among healthcare professionals regarding end-of-life (EOL) care for PwDs; and inadequate caregiver resources to provide care for the PwDs to die at home or to participate in EOL care in a nursing home setting, with the goal of achieving a dignified death.

To address some of these challenges and barriers, our dementia service at G-RACE (Geriatric psychiatry out-Reach Assessment Consultation and Enablement) designed three caregiver workshops for the three stages of dementia according to the third edition of the Diagnostic and Statistical Manual of Mental Disorders from the American Psychiatric Association, or DSM-III-R, with each workshop addressing different care needs pertaining to each stage of dementia. Workshop One covers topics on general health maintenance through control of vascular risk factors and lifestyle changes, introduction to advance care planning and legal matters such as lasting power of attorney, communication strategies, cognitive activities to slow dementia-related decline, as well as community resources. Workshop Two provides education to caregivers regarding neuropsychiatric symptoms that often present in the moderate to severe stage, understanding that some of these symptoms are the PwD's attempts at communicating needs; strategies to manage these symptoms, including nonpharmacological and pharmacological approaches; and an introduction to dementia palliative care and 'Namaste Care'. Workshop Three covers EOL care, addressing individualised goals of care in the bio-psycho-social and spiritual domains, providing available evidence for management of pain, breathlessness, anxiety, agitation and nutrition at the EOL of a PwD.

By inviting PwDs and their caregivers to these workshops from the point of diagnosis, and through the person-centred care that our multidisciplinary team provides over the course of illness and journeying alongside them, we build trust and rapport with the PwDs and the caregivers. This facilitates timely discussion and planning of palliative care, as well as addressing the sensitive spiritual and emotional needs of the PwDs and their families through the course of dementia.

Yours sincerely,

Kar Cheng Goh

G-RACE (Geriatric psychiatry out-Reach Assessment Consultation and Enablement), Department of Psychological Medicine, National University Hospital, 5 Lower Kent Ridge Road, Singapore 119074. kar_cheng_goh@nuhs.edu.sg

\section{References}

1. Tan LL, Ng WF, Sim ACC, Rahim SB. Adopting a palliative approach to dementia care in the community: participatory action research. Singapore Med J 2021; 62:240-3.

2. Department of Statistics, Singapore. Population Trends 2019. https://www.singstat.gov.sg/-/media/files/publications/population/population2019.pdf. Accessed October $21,2021$.

3. Institute of Mental Health, Singapore. Well-Being of the Singapore Elderly (WISE) Study 2013. Available at: https://www.imh.com.sg/research/page.aspx?id=342. Accessed October 21, 2021.

4. Dementia Innovation Readiness Index 2020 - Global Coalition on Aging, Alzheimer's Disease International, Lien Foundation. Available at: https://www.lienfoundation. org/project/dementia-innovation-readiness-index-. Accessed October 21, 2021.

5. Eisenmann Y, Golla H, Schmidt H, Voltz R, Perrar KM. Palliative care in advanced dementia. Front Psychiatry 2020; 11:699.

Editor's note: The authors, Tan et al, have declined to respond to the above letter. 